\title{
Encapsulation versus Aggregation of Metal-Organic Cages Controlled by Guest Size Variation
}

\author{
Guido H. Clever, ${ }^{\dagger}$ Wataru Kawamura, and Mitsuhiko Shionoya* \\ Department of Chemistry, Graduate School of Science, The University of Tokyo, 7-3-1 Hongo, Bunkyo-ku, Tokyo 113-0033, Japan
}

Supporting Information

ABSTRACT: The strength and mode of binding (inside vs outside) of bisanionic guest molecules to a cationic, selfassembled metal-organic cage depend on their size and the stoichiometry of the addition. ${ }^{1}$ Herein we show that the composition of the solid/liquid phase of a heterogeneous system can be kinetically controlled by the order of the addition of two different guest compounds.

Cupramolecular compounds ${ }^{2}$ based on noncovalent interac$\checkmark$ tions promise to find future application as intelligent materials with designed macroscopic properties such as magnetism, conductivity, catalytic activity, porosity, and the capability of storing and releasing small molecules. ${ }^{3}$ Among the various systems developed so far, discrete nanosized cages and capsules are of particular interest for the implementation of specific functions because they provide an inner cavity that can be filled with functional guest molecules when brought together in solution. ${ }^{4}$ Furthermore, intermolecular binding between individual spherical nanocages via their outer surfaces can lead to extended assemblies and bulk materials with interesting properties.

Starting from supramolecular cage $\mathbf{1}$, we here present how the formation of either a discrete, soluble host-guest complex or an insoluble, polymeric material ${ }^{5}$ can be controlled by employing a range of guest molecules that differ in their molecular size. A distinct feature of this system is that, depending on the order of the addition of different guest molecules, the composition of the solid/liquid phases can be controlled as a consequence of the kinetic aspect of the precipitation process (Figure 1).

Cage 1 consists of two $\mathrm{Pd}^{\mathrm{II}}$ ions that are held at a fixed distance of $1.7 \mathrm{~nm}$ by four rigid, concave ligands (Chart 1). ${ }^{6}$ The $\mathrm{Pd}^{\mathrm{II}}$ centers in the square-planar geometry are coordinatively saturated but allow electrostatic interactions of counteranions close to their apical positions both inside and outside the cage to reach charge neutralization. ${ }^{7}$ Previously, we have shown that aromatic bis(sulfonate) anions of appropriate size are quantitatively encapsulated inside the cage, leading to a diagnostic downfield shift of the cage's inside pointing hydrogen atoms $\mathrm{H}_{\mathrm{i}}$ (blue in Chart 1 and Figure 2a) upon facing the sulfonate groups of the guest molecule. ${ }^{6}$ In a recent work, we described the versatility of this binding principle in supramolecular construction by applying it to the formation of $\mathrm{pH}$-switchable (pseudo-)rotaxanes consisting of cage 1 and rodlike bis(sulfonate) guest compounds. ${ }^{8}$ Furthermore, by using a combination of cage $\mathbf{1}$ with a light-switchable cis/trans-4, $4^{\prime}$-azobenzenebis(sulfonate) guest, we realized a hostguest system capable of light-initiated crystallization. ${ }^{9}$ The latter effect was attributed to the change of the guest's molecular dimensions upon irradiation and prompted us to perform a systematic investigation of the influence of the bis(sulfonate) guest's size (in terms of their $S-S$ distance) on the encapsulation and aggregation processes. ${ }^{10}$ We therefore examined the encapsulation of aromatic bis(sulfonate)s $\mathbf{2 - 8}$ depicted in Chart 1, which form a series of planar guests of different size but comparable chemical behavior. NMR titration experiments were used to compare the effect of guest addition on the chemical shift of the characteristic cage proton signals, and a systematic guest displacement assay allowed the qualitative comparison of the relative binding strengths among bis(sulfonate)s $\mathbf{2 - 8}$.

Here, we show that the strength and position (inside/outside) of guest binding depend solely on the guest length and the amount of guest added to the system. The addition of 1 equiv of any bisanionic guest (except 8) to cage 1a resulted in the quantitative formation of a soluble host-guest complex as seen by NMR titration experiments and electrospray ionization (ESI) mass spectrometry. The further addition of excess guest leads to the immediate precipitation of the sample and to a vanishing of the NMR signals (Figures $1 \mathrm{a}$ and $2 \mathrm{a}$ and Supporting Information). To verify our assumption that the outside binding of excess guest is responsible for the precipitation due to aggregation of the cages, we repeated the titration with cage derivative $\mathbf{1 b}$ carrying poly(ethylene glycol) residues attached to each of the eight coordinating pyridine rings, thereby strongly enhancing the solubility of the cages even when an excess amount of guest molecules is added (Figures $1 \mathrm{~b}$ and $2 \mathrm{~b}$ ). Indeed, the addition of the first 1 equiv of guest 7 to cage $1 \mathbf{b}$ leads to quantitative encapsulation, as seen in the case of cage 1a. The presence of excess amounts of 7, however, leads to a downfield shift of the outside pointing hydrogen's NMR signal $\mathrm{H}_{\mathrm{o}}$, with all other cage signals remaining unchanged (purple in Chart 1 and Figure $2 \mathrm{~b}$ ). In our view, this is a clear sign of the excess guest molecules binding to the outside face of the cages, and it is in well accordance with our previous findings using a light-switchable cis/trans-4, $4^{\prime}$-azobenzenebis(sulfonate) guest. ${ }^{9}$ It is noteworthy that "oversized" guest $\mathbf{8}$, which is not able to be fully encapsulated inside cage $\mathbf{1 a} / \mathbf{1} \mathbf{b}$, immediately gives rise to observation of the outside binding mode (see the Supporting Information).

Furthermore, in the case of encapsulation, we found that the NMR shift of the inward pointing hydrogen atom $\mathrm{H}_{\mathrm{i}}$ depends on the size of the bisanionic guest molecules, with increasing guest

Received: March 12, 2011

Published: April 22, 2011 
a)

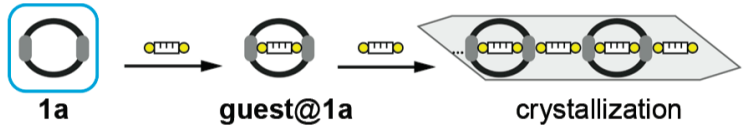

b)

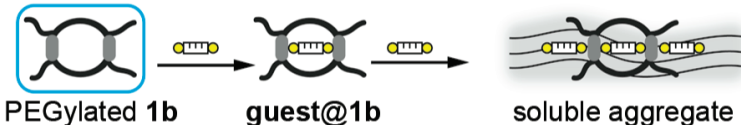

c)

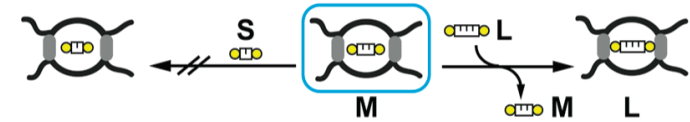

d)

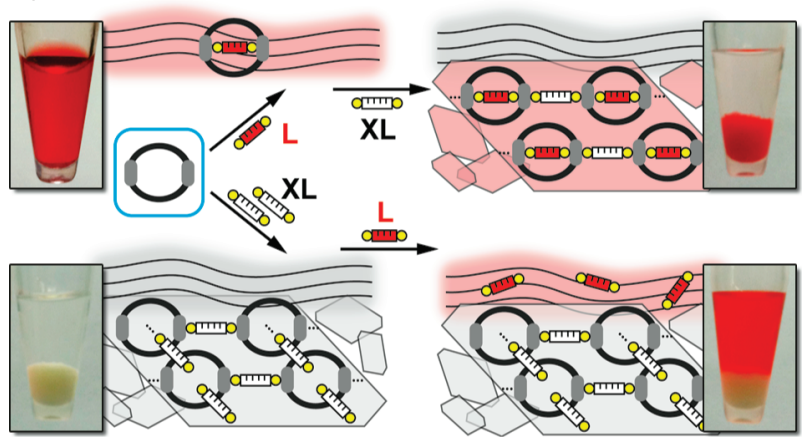

Figure 1. (a) The first guest equivalent is completely encapsulated inside cage 1a, while excess guests lead to immediate precipitation. (b) PEGylated cage $\mathbf{1 b}$ does not precipitate and allows one to study the outside binding of the guests. (c) A smaller guest can be replaced from the cage only by the addition of a longer guest. (d, top) The addition of red guest 9 to cage 1a results in a red solution of the host-guest complex; the subsequent addition of the oversized guest 8 results in complete precipitation of $[(\mathbf{9} @ \mathbf{1 a}) \mathbf{8}]$ and a colorless supernatant. (d, bottom) The first addition of 8 leads to precipitation; the addition of 9 to the suspension of $\left[(\mathbf{1 a})(8)_{2}\right]_{n}$ results in a red solution with a colorless precipitate. $S=$ short, $M=$ medium, $\mathrm{L}=$ long size.

Chart 1. Structures of Cages 1a and $1 \mathrm{~b}$ and Bis(sulfonate) Guests 2-9

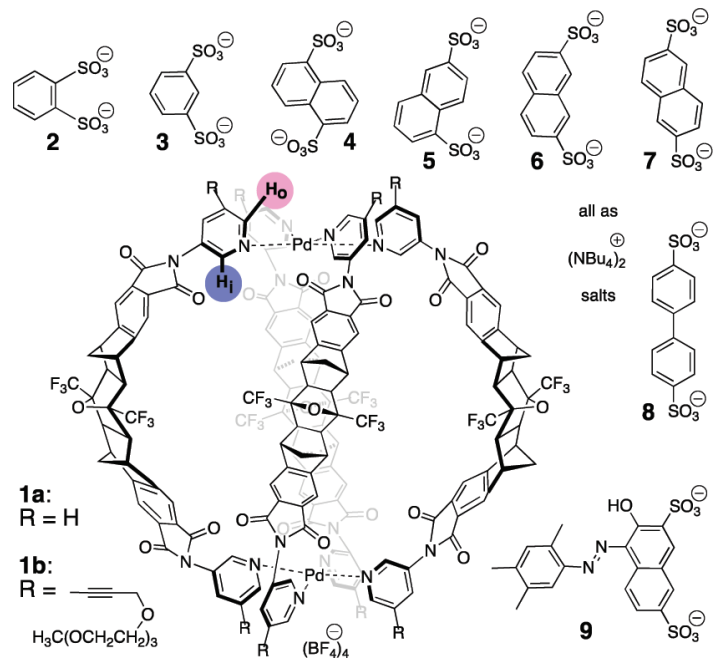

size leading to increasing downfield shifts (Figure 2c). ${ }^{11}$ We denote this observation to a decrease in the sulfonate $-\mathrm{H}_{\mathrm{i}}$ distances with (a) pure guest 7

(b) pure guest 7,

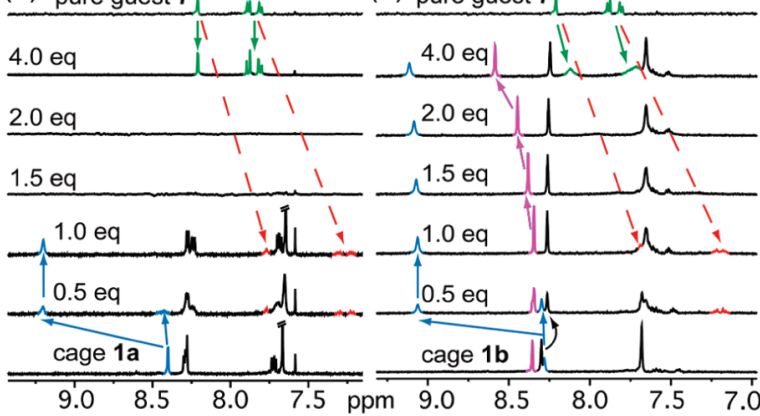

(c)
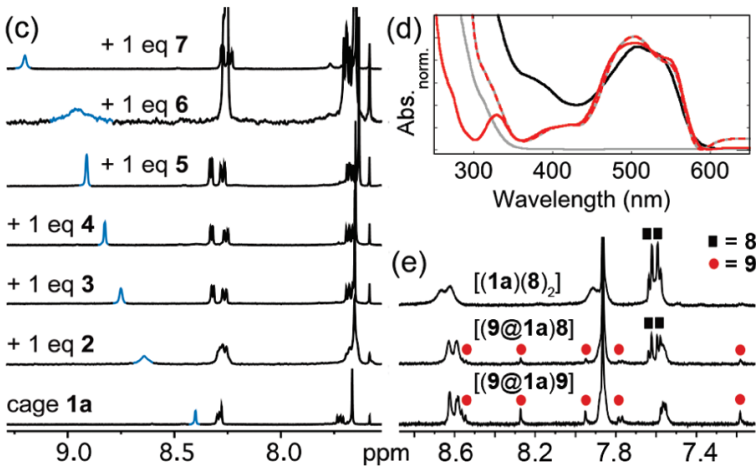

(e)

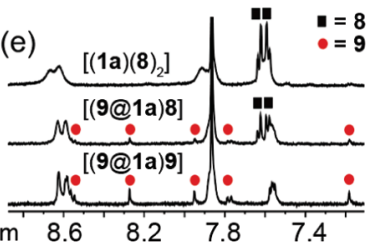

Figure 2. (a) The titration of cage 1a with 7 shows the quantitative uptake of up to 1 equiv of the guest and precipitation of the cages upon the addition of excess guest. (b) A similar titration of $\mathbf{1 b}$ with 7 shows the outside binding of excess guest to the cages. (c) The NMR spectra of the host-guest complexes with 2-7 show an increasing shift of the signal of the inward pointing hydrogen atom with increasing guest size. (d) ATRUV spectra of solid samples $\left[(\mathbf{1 a})(\mathbf{8})_{2}\right]$ (gray), 9 (red), $\left[(\mathbf{1 a})(\mathbf{8})_{2}\right]+\mathbf{9}$ (red dashed), and [(9@1a)8] (black) in a KBr matrix. (e) NMR analysis of the precipitate compositions in DMSO- $d_{6}$ shows the expected relative amounts of guests $\mathbf{9}$ and $\mathbf{8}$ and cage components.

increasing guest size inside the rigid cage structure. The strength of binding inside the cage increases in the same direction as shown qualitatively by a systematic study of guest replacement experiments (Figure 1c, Table 1, and Supporting Information).

Next, we combined our findings about the roles of the guest size, amount, and binding mode to design a simple multicomponent system in which the onset of precipitation and the composition of the solid and liquid phases can be controlled by the order of the addition of selected guest molecules (Figure 1d). In order to make the behavior of the system visible to the naked eye, we chose red dye 9 (also known as Japan Red 1 or Ponceau-3R) as a derivative of guest 6 to be incorporated inside the cage. Upon the addition of 1 equiv of 9 to the cage, a clear, red solution is formed (Figure 1d, top). The formation of [9@1a] was confirmed by ${ }^{1} \mathrm{H}$ NMR and ESI mass spectrometry (see the Supporting Information). The addition of 1 equiv of colorless, oversized guest 8 resulted in the formation of a red precipitate $[(9 @ 1 \mathbf{a}) 8]$ and an almost colorless supernatant void of any cage or guest NMR signals. ${ }^{12}$

In contrast, when $\mathbf{8}$ was added first to the cage solution, the immediate formation of a colorless precipitate $\left[(\mathbf{1 a})(8)_{2}\right]$ took place (Figure 1d, bottom). A total of 2 equiv of 8 was needed to complete precipitation in this case. We assume that compound 8 can replace also the interior $\mathrm{BF}_{4}{ }^{-}$ions by reaching partly inside the cages through their large openings without being able to be completely incorporated. ${ }^{13}$ The addition of red guest 9 to this sample leads to a red solution above an almost colorless precipitate. 
Table 1. NMR-Based Guest Replacement Experiments with Cage $1 b^{a}$

$\begin{array}{ccccc} & 3 & 4 & 6 & 7 \\ 3 & & - & - & - \\ 4 & \mathrm{X} & & \mathrm{a} & - \\ 6 & \mathrm{X} & \mathrm{a} & & - \\ 7 & \mathrm{X} & \mathrm{X} & \mathrm{X} & \end{array}$

${ }^{a}$ Horizontal row: guests first added to cage $\mathbf{1 b}$. Vertical row: guests added subsequently. $\mathrm{X}$ : complete guest displacement. -: no guest displacement. a: NMR spectra showing the formation of a mixture of 4@1b and6@1b.

The immediate formation of the precipitates yielded a microcrystalline material, as was clearly observed by light microscopy. Characterization by X-ray analysis, however, failed because of the small size and possibly the high solvent content of the obtained crystals. Insights into the composition and structure, however, could be deduced from the following methods. A comparison of the ATR$U^{14}$ spectra of solid samples of the free red dye 9 with the precipitate [(9@1a)8] showed a greatly enhanced absorption around $380 \mathrm{~nm}$ for the host-guest complex, which is in accordance with the UV spectroscopic results obtained for the solutions of free guest 9 and the host-guest complex [9@1a] in $\mathrm{CH}_{3} \mathrm{CN}$ (see the Supporting Information), thereby indicating guest 9 to be encapsulated by cage 1a in the red precipitate [(9@1a)8]. This effect is not observed when solid samples of $\mathbf{9}$ and $\left[(\mathbf{1 a})(\mathbf{8})_{2}\right]$ are mixed in the dry $\mathrm{KBr}$ matrix just prior to the measurement (Figure $2 \mathrm{~d}$ ). In the latter case, encapsulation of 9 by cage $\mathbf{1 a}$ is not feasible in the diluted powder sample and the ATR-UV spectrum can be interpreted as an additive spectrum composed of contributions from the spectra of the individual samples $\left[(\mathbf{1 a})(\mathbf{8})_{2}\right]$ and 9 .

Furthermore, the precipitates $\left[(\mathbf{1 a})(\mathbf{8})_{2}\right]$ and $[(\mathbf{9} @ \mathbf{1 a}) \mathbf{8}]$ as well as the precipitate [(9@1a)9] (which is formed when 2 equiv of guest 9 is added to a solution of cage 1a) could be dissolved in hot dimethyl sulfoxide (DMSO)- $d_{6}$, and their proposed composition was confirmed by NMR spectroscopy (Figure 2e and Supporting Information).

In conclusion, we could show how replacement of the monovalent counteranions from a rigid molecular cage by aromatic bisanions can be controlled to form discrete host-guest complexes or precipitates by careful choice of the kind, amount, and order of the addition of guest compounds. We think this strategy may contribute to the toolbox of supramolecular architecture and enable the generation of more complex structures and materials, e.g., by the use of further functionalized guest molecules.

\section{ASSOCIATED CONTENT}

S Supporting Information. Experimental details and further NMR, UV, and mass spectra. This material is available free of charge via the Internet at http://pubs.acs.org.

\section{AUTHOR INFORMATION}

\section{Corresponding Author}

*E-mail: shionoya@chem.s.u-tokyo.ac.jp.

\section{Present Addresses}

${ }^{\dagger}$ Institut für Anorganische Chemie, Georg-August-Universität Göttingen, Tammannstrasse 4, 37077 Göttingen, Germany.

\section{ACKNOWLEDGMENT}

This work was funded by the Global COE Program for Chemistry Innovation, a JSPS start-up grant, a JSPS postdoctoral scholarship, and a fellowship of the Alexander von Humboldt Foundation for G.H.C.

\section{REFERENCES}

(1) For the sake of clarity, we use the term "guest" here for all discussed bisanionic compounds regardless of whether they are actually found inside the cages or associated with the outer sides of the cages.

(2) Steed, J. W.; Atwood, J. L. Supramolecular Chemistry, 2nd ed.; John Wiley \& Sons: New York, 2009.

(3) (a) Simon, J.; Bassoul, P. Design of Molecular Materials; John Wiley \& Sons: New York, 2001. (b) Ozin, G. A.; Arsenault, A. C.; Cademartiri, L. Nanochemistry, 2nd ed.; RSC Publishing: Cambridge, U.K., 2009.

(4) For reviews see:(a) Dalgarno, S. J.; Power, N. P.; Atwood, J. L. Coord. Chem. Rev. 2008, 252, 825. (b) Tranchemontagne, D. J. L.; Ni, Z.; O'Keeffe, M.; Yaghi, O. M. Angew. Chem., Int. Ed. 2008, 47, 5136. (c) Vriezema, D. M.; Aragones, M. C.; Elemans, J. A. A. W.; Cornelissen, J.J. L. M.; Rowan, A. E.; Nolte, R. J. M. Chem. Rev. 2005, 105, 1445.

(5) Brunsveld, L.; Folmer, B. J.; Meijer, E. W.; Sijbesma, R. P. Chem. Rev. 2001, 101, 4071.

(6) Clever, G. H.; Tashiro, S.; Shionoya, M. Angew. Chem., Int. Ed. 2009, 48, 7010 .

(7) Several other groups have reported coordination cages of the general formula $\left[\mathrm{M}_{2} \mathrm{~L}_{4}\right]^{4+}$, where $\mathrm{M}$ is a square-planar-coordinated metal ion such as $\mathrm{Pd}^{\mathrm{II}}$ or $\mathrm{Pt}^{\mathrm{II}}$ and $\mathrm{L}$ are bis(pyridyl) ligands bridging the two metal centers. These cages, however, are much smaller and only offer limited potential for expecting a rich host-guest chemistry. For example, see: (a) McMorran, D. A.; Steel, P. J. Angew. Chem., Int. Ed. 1998, 37, 3295. (b) Chand, D. K.; Biradha, K.; Fujita, M. Chem. Commun. 2001, 1652. (c) Su, C.; Cai, Y. P.; Chen, C.; Smith, M. D.; Kaim, W.; zur Loye, H. C. J. Am. Chem. Soc. 2003, 125, 8595. (d) Yue, N. L. S.; Eisler, D. J.; Jennings, M. C.; Puddephatt, R. J. Inorg. Chem. 2004, 43, 7671. (e) Fukuda, M.; Sekiya, R.; Kuroda, R. Angew. Chem., Int. Ed. 2008, 47, 706. (f) Liao, P.; Langloss, B. W.; Johnson, A. M.; Knudsen, E. R.; Tham, F. S.; Julian, R. R.; Hooley, R. J. Chem. Commun. 2010, 4932.

(8) Clever, G. H.; Shionoya, M. Chem.-Eur. J. 2010, 16, 11792.

(9) Clever, G. H.; Tashiro, S.; Shionoya, M. J. Am. Chem. Soc. 2010, 132, 9973.

(10) Sessler, J. L.; Gale, P. A.; Cho, W.-S. Anion Receptor Chemistry; RSC Publishing: Cambridge, U.K., 2006.

(11) Hiraoka, S.; Kiyokawa, M.; Hashida, S.; Shionoya, M. Angew. Chem., Int. Ed. 2009, 49, 138.

(12) Except for the ${ }^{1} \mathrm{H}$ NMR signals of the tetrabutylammonium counterions.

(13) The results obtained by using guest 9 are in perfect agreement with the observations that we made when using trans-4,4'azobenzenebis(sulfonate) as a guest (see ref 7). Both compounds are of comparable size in terms of the $\mathrm{S}-\mathrm{S}$ distance. Molecular modeling reveals that both compounds are too large to be fully incorporated inside cage 1 in the fashion of a 1:1 host-guest compound. Instead, it was found that a crystalline supramolecular polymer of composition host: guest $=1: 2$ is formed, with one molecule of trans- $4,4^{\prime}$-azobenzenebis(sulfonate) joining the cages by electrostatic binding to the outer faces of the $\mathrm{Pd}(\mathrm{py})_{4}$ complex planes and 1 equiv cross-linking these neighboring (cage- guest $)_{\mathrm{n}}$ chains by partial penetration into two cages.

(14) ATR (attenuated total reflection)-UV spectra were recorded in a $\mathrm{KBr}$ matrix and background-corrected. 